Chirurg 2012 $\cdot 83: 857$

DOI 10.1007/s00104-012-2336-4

Online publiziert: 27. September 2012

c) Springer-Verlag Berlin Heidelberg 2012

\title{
C. Josten
}

Klinik und Poliklinik für Unfall-, Wiederherstellungs- und Plastische Chirurgie,

Wirbelsäulenzentrum, Universitätsklinik Leipzig

\section{Osteoporotische Frakturen}

\section{Was ist neu?}

Obwohl die idiopathische Osteoporose auf einen physiologischen Alterungsprozess zurückzuführen ist, wird sie zwischenzeitlich als Alterserkrankung bezeichnet. Mit 95\% am häufigsten ist die primäre Osteoporose; fast $80 \%$ der Frauen sind davon betroffen. Sekundäre Osteoporosen sind deutlich seltener, wobei hier eine begleitende Behandlung mit Kortikoiden über einen längeren Zeitraum in Vordergrund steht.

Zur Diagnose und Behandlung der Osteoporose liegen feste Leitlinien vor, nach denen jeder Patient mit einer entsprechenden Fraktur untersucht werden sollte, was jedoch nur in weniger als der Hälfte der Fälle geschieht. Dies ist insbesondere von hoher Bedeutung, da von den fast 8 Mio. Osteoporosepatienten jedes Jahr 4-5\% eine derartige Verletzung erleiden.

\section{》) Osteoporose ist eine kostenintensive Volkskrankheit}

Die Osteoporose kann als eine bedeutende Volkskrankheit betrachtet werden, denn fast jeder 10. Deutsche ist davon betroffen, und etwa 500.000 Frakturen sind jährlich auf eine Osteoporose zurückzuführen. Der Knochenbruch ist zumeist das erste Zeichen einer Osteoporose, aber es werden z. B. fast $30 \%$ der Wirbelfrakturen primär nicht erkannt. Nur eine umfassende Diagnostik und korrekte Operationstechnik unter Anwendung minimalinvasiver Techniken und moderner Im- plantate gewährleisten einen möglichst risikoarmen Eingriff, eine geringe postoperative Morbidität sowie ausreichende Stabilität und Belastbarkeit der Fraktur. Trotzdem liegt die Komplikationsrate osteoporotischer Frakturen deutlich höher als bei jungen Patienten ohne Osteoporose.

Die Krankheitskosten der Osteoporose werden auf insgesamt 5,4 Mrd. EUR pro Jahr geschätzt, wobei für die Versorgung von Knochenbrüchen schon über $60 \%$ (3,3 Mrd. EUR) aufgewendet werden müssen. Damit gehört die Osteoporose zu den kostenintensiveren Volkskrankheiten.

Typische osteoporotische Frakturen sind, nach Häufigkeit aufgeführt, folgende:

- Wirbelkörperfrakturen,

- hüftgelenksnahe Oberschenkelfrakturen,

- distale Radiusfrakturen,

- subkapitale und kapitale Humerusfrakturen,

- Beckenfrakturen.

In den folgenden Arbeiten haben wir uns den häufigsten osteoporosebedingten Frakturen angenommen und wollen den heutigen Stand in der Diagnostik und Therapie aufzeigen, aber auch die zahlreichen Möglichkeiten der Komplikationen.

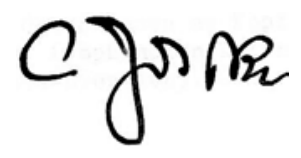

Prof. Dr. C. Josten

\section{Korrespondenzadresse}

Prof. Dr. C. Josten

Klinik und Poliklinik für Unfall-, Wiederherstellungs- und Plastische Chirurgie, Wirbelsäulenzentrum, Universitätsklinik Leipzig, Liebigstr. 20, 04103 Leipzig

Christoph.Josten@medizin.uni-leipzig.de 